\title{
Pyridoxal 5'-phosphate-responsive epilepsy with novel mutations in the PNPO gene: a case report
}

\author{
M. Veeravigrom ${ }^{1,2}$, P. Damrongphol ${ }^{1,2}$, R. Ittiwut ${ }^{3,4}$, C. Ittiwut ${ }^{3,4}$, \\ K. Suphapeetiporn ${ }^{3,4}$ and V. Shotelersuk ${ }^{3,4}$ \\ 1Division of Neurology, Department of Pediatrics, Faculty of Medicine, \\ Chulalongkorn University, Thailand \\ 2Division of Neurology, Department of Pediatrics, \\ King Chulalongkorn Memorial Hospital, The Thai Red Cross Society, \\ Bangkok, Thailand \\ ${ }^{3}$ Center of Excellence for Medical Genetics, Department of Pediatrics, \\ Faculty of Medicine, Chulalongkorn University, Bangkok, Thailand \\ ${ }^{4}$ Excellence Center for Medical Genetics, King Chulalongkorn Memorial Hospital, \\ The Thai Red Cross Society, Bangkok, Thailand \\ Corresponding author: K. Suphapeetiporn \\ E-mail: kanya.su@chula.ac.th
}

Genet. Mol. Res. 14 (4): 14130-14135 (2015)

Received June 2, 2015

Accepted September 1, 2015

Published October 29, 2015

DOI http://dx.doi.org/10.4238/2015.October.29.34

ABSTRACT. Pyridoxal 5'-phosphate (PLP)-responsive epilepsy is a rare autosomal recessive epileptic disorder caused by deficiency of pyridox(am)ne 5'-phosphate oxidase (PNPO). Neonatal onset seizures in PLP responsive epilepsy are usually resistant to common anticonvulsants and pyridoxine, but respond to PLP. Various PNPO mutations are associated with this disorder. In this report, we have described a case of a female baby with neonatal onset seizures responding to PLP. Exome sequencing revealed that the patient was compound heterozygous for pathogenic mutations [c.546+1G>A (IVS5+1 G>A) and c.620delG (p.G207VfsX215)] 
in the PNPO gene. The c.546+1G>A was inherited from the mother while the c.620delG was inherited from the father. Both mutations were absent in 122 unrelated Thai controls. The results of this study indicated the presence of two newly identified mutations in this Thai patient with PLP-responsive epilepsy for the first time, expanding the mutational spectrum of PNPO.

Key words: Epilepsy; Pyridoxal 5'-phosphate; Mutation; Pyridox(am)ine 5'-phosphate oxidase

\section{INTRODUCTION}

Pyridoxal 5'-phosphate (PLP)-responsive epilepsy or pyridox(am)ine 5'-phosphate oxidase (PNPO) deficiency is a rare autosomal recessive disorder caused by deficiency of PNPO (OMIM 610090). The PNPO enzyme is a key factor for conversion of pyridoxine and pyridoxamine into PLP. PLP is an important vitamin B6 cofactor for synthesis of neurotransmitters, especially gamma-aminobutyric acid (GABA). Seizures in PLP-responsive epilepsy are resistant to common anticonvulsants and pyridoxine (Mills et al., 2005).

The PNPO gene contains seven exons, and encodes a 261-amino acid protein. At least 24 different disease-causing mutations scattered throughout the PNPO gene have been reported, with the most common being missense/nonsense mutations (Human Gene Mutation Database, http://www.hgmd.cf.ac.uk, accessed April 2015). In this study, we have reported a Thai female infant with PLP-responsive epilepsy. Two novel mutations in the PNPO gene, including a splicing mutation and a single-base pair deletion, were identified.

\section{MATERIAL AND METHODS}

\section{Patient information}

The female infant (24 days old) was referred to King Chulalongkorn Memorial Hospital. The patient was the second child of non-consanguineous Thai parents. The pregnancy was complicated, with intrauterine growth restriction and oligohydramnios. The patient was born prematurely at 32 weeks gestation; the birth weight of the patient was $2305 \mathrm{~g}$, and her Apgar scores at 1 and 5 min were 9 and 9 , respectively. She developed respiratory distress and was diagnosed with respiratory distress syndrome. She exhibited symptoms of myoclonic seizures $6 \mathrm{~h}$ after birth. A full-septic work-up and metabolic investigations were performed with unremarkable results. The patient was treated with phenobarbital, which was slowly titrated to $9 \mathrm{mg} \cdot \mathrm{kg}^{-1} \cdot \mathrm{day}^{-1}$.

At the age of 10 days, the patient underwent several episodes of myoclonic seizures. Phenytoin was added at a maintenance dose of $7 \mathrm{mg} \cdot \mathrm{kg}^{-1} \cdot \mathrm{day}^{-1}$. Subsequently, the frequency of myoclonic seizures was reduced to 1-2 times per day. The patient was then discharged at the age of 21 days. Two days after the hospital discharge, the patient experienced multiple clusters of multifocal and generalized myoclonic seizures, intermixed with eye deviation and inconsolable crying, which developed into myoclonic status epilepticus. A computed tomography (CT) scan of the brain showed cerebral edema with diffused, non-specific, poor gray-white 
matter differentiation; subsequently, assisted ventilation, and a regimen of cefotaxime and acyclovir was initiated. The epilepticus status of the patient was refractory to multiple-antiepileptic medications, including phenytoin, phenobarbital, levetiracetam, and sodium valproate. A trial of intravenous pyridoxine did not stop the clinical seizure. Continuous midazolam infusion was administered and titrated up to $40 \mu \mathrm{g} \cdot \mathrm{kg}^{-1} \cdot \mathrm{min}^{-1}$. The patient was then referred to our hospital. Cerebral function monitoring during high-dose midazolam infusion showed a burst-suppression pattern. A $10 \mathrm{mg} / \mathrm{kg}$ PLP trial resulted in an increased suppression ratio and flattening of the electroencephalogram (EEG). A brain CT scan revealed no other causes of electrocerebral silence. The patient brain showed normal results when subjected to magnetic resonance imaging and magnetic resonance spectrometry; in addition, her urine organic acid screening was unremarkable. Discontinuation of the midazolam treatment resulted in a gradual return of continuous EEG pattern and the patient regaining consciousness. Because of her dramatic response to PLP, PLP-responsive epilepsy was suspected. The maintenance of a PLP dose of $30 \mathrm{mg} \cdot \mathrm{kg}^{-1} \cdot \mathrm{day}^{-1}$ helped control the seizures. Sodium valproate was tapered off by the time the patient turned 2 months old. The patient remained seizure-free when subjected to a constant dosage of $6 \mathrm{mg} \cdot \mathrm{kg}^{-1} \cdot \mathrm{day}^{-1}$ phenytoin, $4 \mathrm{mg} \cdot \mathrm{kg}^{-1} \cdot \mathrm{day}^{-1}$ phenobarbital, and $20 \mathrm{mg}$. $\mathrm{kg}^{-1} \cdot$ day $^{-1}$ levetiracetam, in addition to $30 \mathrm{mg} \cdot \mathrm{kg}^{-1} \cdot \mathrm{day}^{-1} \mathrm{PLP}$.

\section{Mutation analysis}

Mutation analysis was performed by whole exome sequencing (WES). This study was considered to be exempt from review by the Institutional Review Board of the Faculty of Medicine, Chulalongkorn University. Informed consent was obtained from the parents, and peripheral blood samples were collected from the patient and her parents. Genomic DNA was extracted from the peripheral blood leukocytes of the patient using the Gentra Puregene Blood Kit (Qiagen, Valencia, CA, USA), and sent to Macrogen Inc. (Seoul, Korea) for WES.

The primers for mutation validation were designed using the Primer3 software. The primers used to detect the c.546+1G>A and c.620delG mutations in the PNPO gene were 5'-CAGAGCCATCCCTGAGCAG-3' and 5'-CACTTTCCCTTCTCACTGTCCT-3', and 5'-CCCTTGTCAGATGCATCCCA-3' and 5'-AGGTCCCAGAGTTAAGGTGC-3', respectively. The PCR products were treated with ExoSAP-IT (USP Corporation, Cleveland, OH, USA) and sent for direct sequencing to Macrogen. The sequence data were analyzed using the Mutation Surveyor software (Softgenetics, State College, PA, USA).

\section{RESULTS}

Two different novel pathogenic mutations were discovered in the PNPO gene. The patient displayed compound heterozygosity for the c.546+1G>A (IVS5+1 G>A) and c.620delG (p.G207VfsX215) located in exon 7. Both were validated by Sanger sequencing of the genomic DNA obtained from the patient and her parents (Figure 1). The splicing mutation c.546+1G>A was inherited from the mother, while the single-base pair deletion causing a frameshift (c.620delG) was inherited from the father. Both mutations were absent in 122 unrelated Thai exome controls. 


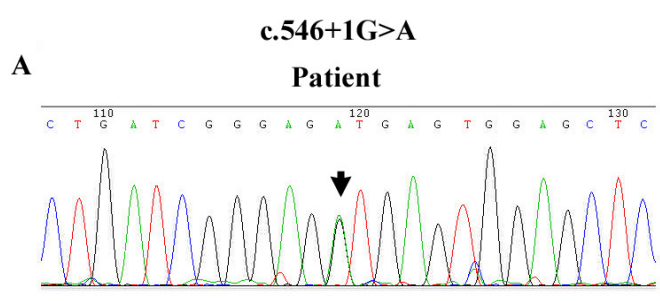

Mother

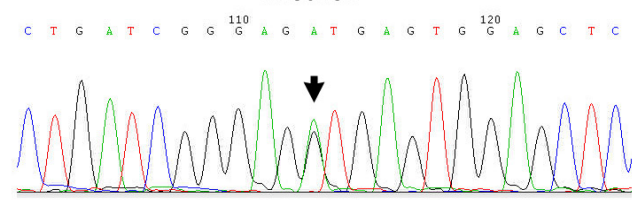

Father

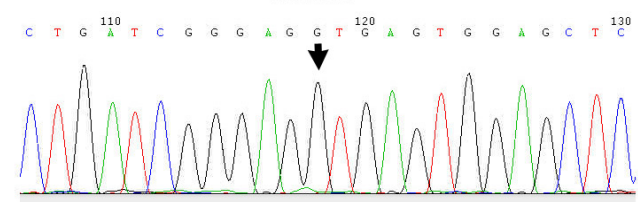

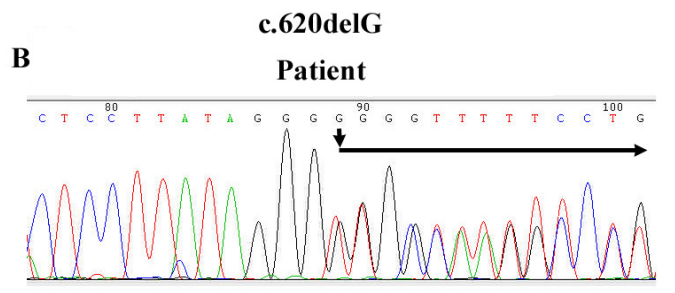

Mother

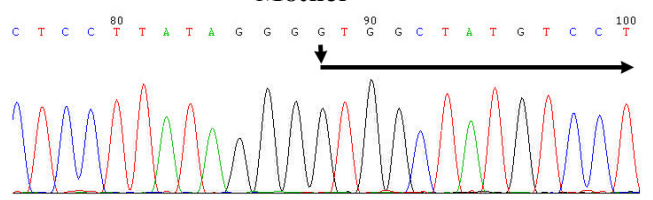

Father

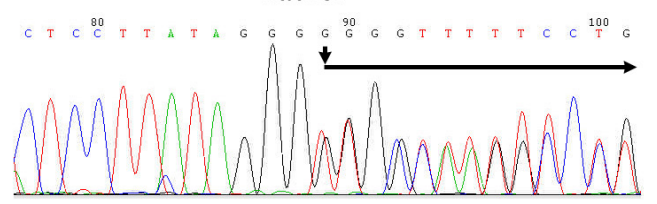

Figure 1. Electropherograms of the patient and both parents. A. The c.546+1G>A (IVS5+1 G>A) mutation is inherited from the mother. B. The c.620delG (p.G207VfsX215) mutation is inherited from the father. Each mutation is indicated by an arrow.

\section{DISCUSSION}

In addition to its rarity, the non-specific clinical features and EEG patterns increase the difficulty of early recognition of PLP-responsive epilepsy. Our patient experienced neonatal onset seizures refractory to anticonvulsant medications. The discovery that the seizures responded to PLP and not pyridoxine prompted the investigation of the genetic defects in the PNPO gene. Exome sequencing, followed by targeted Sanger sequencing, revealed two novel causative mutations in the proband, each of which was inherited from each parent.

PLP-responsive epilepsy is highly correlated with the incidence of prematurity (Mills et al., 2014; Plecko et al., 2014). Seizures resulting from PNPO deficiency typically occur in utero, or shortly after birth, with various, ictal manifestations predominantly multifocal and generalized myoclonic seizures that are refractory to conventional anticonvulsants and pyridoxine. Our patient was born prematurely, and the mother did not report any rhythmic fetal movements. The patient developed seizures $6 \mathrm{~h}$ after birth, exhibited a transient response to phenobarbital and phenytoin, and subsequently progressed to myoclonic status epilepticus.

EEG findings in PNPO deficiency are not pathognomonic. A previous study has described a burst suppression pattern in PNPO deficiency mimicking nonketotic hyperglycinemia and Ohtahara syndrome (Mills et al., 2005). Our patient was not subjected to an EEG at the onset of the seizures. At 25 days of age, a supramaximal dose of midazolam resulted in a burst suppression pattern. EEG was transformed into electrocerebral silence after a trial of PLP. In a PNPO deficient state with accumulation of glutamate, administration of PLP would cause the same result as an intravenous pyridoxine infusion in pyridoxine-dependent epilepsy, i.e., electrocerebral suppression, hypotension, and apnea. The exact mechanism of this reaction is unknown; however, it may be 
related to a sudden increase in GABA concentration in the cerebrospinal fluid (CSF) (Bass et al., 1996). This emphasizes the need for close monitoring and preparation for resuscitation after administration of PLP in PNPO deficiency, as well as pyridoxine-dependent epilepsy.

The metabolic investigations in PNPO deficiency characteristically demonstrate low PLP levels in the plasma and CSF, elevated levels of L-3,4-dihydroxyphenylalanine, 3-O-methyldopa, glycine, and threonine in CSF, and increased urinary excretion of very long chain fatty acids (Bass et al., 1996; Mills et al., 2005; Clayton, 2006). Biochemical studies were not performed in this case because of sample unavailability. However, the diagnosis of PNPO deficiency was further confirmed by mutation analysis, which revealed mutations in both alleles of the PNPO gene.

Various clinical courses and outcomes have been reported in previous cases of PNPO deficiency (Plecko, 2013; Plecko et al., 2014). If left untreated, the patients can develop failure to thrive, refractory seizures, and severe encephalopathy, leading to early death. Patients in whom the disease was recognized early, and who received appropriate treatment are believed to be more likely to have favorable outcomes (Hoffmann et al., 2007; Porri et al., 2014). This was supported by the results of a previous study describing a male infant with a homozygous splice-site mutation in the PNPO gene. The patient had seizures within $12 \mathrm{~h}$ after birth, and received PLP treatment on day 3; a favorable neurological outcome was observed at the age of 3 months (Porri et al., 2014). Delay in PLP treatment usually leads to motor and intellectual disabilities. However, some studies have also reported a good outcome in patients with delayed PLP treatment (Hoffmann et al., 2007; Schmitt et al., 2010; Pearl et al., 2013). One patient developed a seizure on the second day after birth, and was administered PLP at 27 days when PNPO deficiency was suspected. A developmental screening test performed at 9 months of age revealed normal findings. This patient was compound heterozygous for a missense mutation and a single-base pair deletion in the PNPO gene (Schmitt et al., 2010). Another patient with a homozygous missense PNPO mutation experienced seizures within $12 \mathrm{~h}$ after birth; this patient was administered with PLP at 3.5 months of age because of breakthrough seizures despite pyridoxine therapy. Subsequent developmental assessment at the age of 28 months showed mild hypotonia and language delay. Different types of mutations in the PNPO gene were theorized to affect the disease severity.

Because of the limited number of cases with PLP-responsive epilepsy confirmed by molecular methods, the genotype-phenotype correlations remain unclear. Our patient was compound heterozygous for the $c .546+1 G>A$ and c.620delG mutations. The c.546+1G $>A$ (IVS5+1 G>A), located in intron 5, was predicted to disrupt the splice site consensus sequence. The splice donor site mutation would cause either aberrant splicing involving exon 5, resulting in a shortened protein product, or nonsense-mediated decay, wherein the entire transcript would be removed to avoid the production of a truncated protein. The c.620delG located in exon 7 was expected to cause a frameshift mutation, leading to premature termination at codon 215 (p.G207VfsX215). This shortened protein would lead to a loss of several amino acids that play an important role in facilitating binding of the flavin mononucleotide to the active site of PNPO (Mills et al., 2005). Previous studies have investigated the effect of the mutations in exon 7 (including p.R225H and p.R229W) on PNPO, discovering a significant reduction in the PNPO activity (Mills et al., 2014).

In conclusion, two novel mutations were successfully identified in the PNPO gene in a Thai family, and the mutational spectrum of PNPO causing PLP-responsive epilepsy was expanded. This study has emphasized the significant implication of genetic testing in PNPO-deficient patients, leading to a more accurate genetic diagnosis and effective genetic counseling. 


\section{Conflicts of interest}

The authors declare no conflict of interest.

\section{ACKNOWLEDGMENTS}

We would like to thank Dr. Tayard Desudchit and Dr. Khemmachart Pongsanon for providing excellent patient care. Research supported by the Thailand Research Fund, the National Science and Technology Development Agency, and the Ratchadaphiseksomphot Endowment Fund of Chulalongkorn University (\#RES560530177-HR).

\section{REFERENCES}

Bass NE, Wyllie E, Cohen B and Joseph SA (1996). Pyridoxine-dependent epilepsy: the need for repeated pyridoxine trials and the risk of severe electrocerebral suppression with intravenous pyridoxine infusion. J. Child. Neurol. 11: $422-424$.

Clayton PT (2006). B6-responsive disorders: a model of vitamin dependency. J. Inherit. Metab. Dis. 29: 317-326.

Hoffmann GF, Schmitt B, Windfuhr M, Wagner N, et al. (2007). Pyridoxal 5'-phosphate may be curative in early-onset epileptic encephalopathy. J. Inherit. Metab. Dis. 30: 96-99.

Mills PB, Surtees RA, Champion MP, Beesley CE, et al. (2005). Neonatal epileptic encephalopathy caused by mutations in the PNPO gene encoding pyridox(am)ine 5'-phosphate oxidase. Hum. Mol. Genet. 14: 1077-1086.

Mills PB, Camuzeaux SS, Footitt EJ, Mills KA, et al. (2014). Epilepsy due to PNPO mutations: genotype, environment and treatment affect presentation and outcome. Brain 137: 1350-1360.

Pearl PL, Hyland K, Chiles J, McGavin CL, et al. (2013). Partial Pyridoxine Responsiveness in PNPO Deficiency. JIMD Rep. 9: $139-142$.

Plecko B (2013). Pyridoxine and pyridoxalphosphate-dependent epilepsies. Handb. Clin. Neurol. 113: 1811-1817.

Plecko B, Paul K, Mills P, Clayton P, et al. (2014). Pyridoxine responsiveness in novel mutations of the PNPO gene. Neurology 82: 1425-1433.

Porri S, Fluss J, Plecko B, Paschke E, et al. (2014). Positive outcome following early diagnosis and treatment of pyridoxal-5'phosphate oxidase deficiency: a case report. Neuropediatrics 45: 64-68.

Schmitt B, Baumgartner M, Mills PB, Clayton PT, et al. (2010). Seizures and paroxysmal events: symptoms pointing to the diagnosis of pyridoxine-dependent epilepsy and pyridoxine phosphate oxidase deficiency. Dev. Med. Child. Neurol. 52: e133-e142. 